\title{
Fractal Method for Modeling the Peculiar Dynamics of Transient Carbon Plasma Generated by Excimer Laser Ablation in Vacuum
}

\author{
C. Ursu, ${ }^{1}$ P. Nica $\mathbb{D}^{2},{ }^{2}$ C. Focsa, ${ }^{3}$ and M. Agop $\mathbb{D D}^{2}$ \\ ${ }^{1}$ Polymer Materials Physics Laboratory, "Petru Poni" Institute of Macromolecular Chemistry, 41 A Gr. Ghica Voda Alley, \\ 700487 Iasi, Romania \\ ${ }^{2}$ Department of Physics, Gheorghe Asachi Technical University, 700050 Iasi, Romania \\ ${ }^{3}$ Univ. Lille, CNRS, UMR 8523, Physique des Lasers, Atomes et Molécules (PhLAM), Centre d'Etudes et de Recherches Lasers et \\ Applications (CERLA), 59000 Lille, France
}

Correspondence should be addressed to P. Nica; pnica@tuiasi.ro

Received 1 March 2018; Accepted 8 July 2018; Published 12 August 2018

Academic Editor: Thach Ngoc Dinh

Copyright (C) 2018 C. Ursu et al. This is an open access article distributed under the Creative Commons Attribution License, which permits unrestricted use, distribution, and reproduction in any medium, provided the original work is properly cited.

Carbon plasmas generated by excimer laser ablation are often applied for deposition (in vacuum or under controlled atmosphere) of high-technological interest nanostructures and thin films. For specific excimer irradiation conditions, these transient plasmas can exhibit peculiar behaviors when probed by fast time- and space-resolved optical and electrical methods. We propose here a fractal approach to simulate this peculiar dynamics. In our model, the complexity of the interactions between the transient plasma particles (in the Euclidean space) is substituted by the nondifferentiability (fractality) of the motion curves of the same particles, but in a fractal space. For plane symmetry and particular boundary conditions, stationary geodesic equations at a fractal scale resolution give a fractal velocity field with components expressed by means of nonlinear solutions (soliton type, kink type, etc.). The theoretical model successfully reproduces the (surprising) formation of V-like radiating plasma structures (consisting of two lateral arms of high optical emissivity and a fast-expanding central part of low emissivity) experimentally observed.

\section{Introduction}

Plasma structures are often assimilated into complex systems, when considering their functionality and structures $[1,2]$. The models commonly used for studying their dynamics are assuming the differential hypothesis of the physical quantities describing space and time evolution, such as energy, momentum, density, and so on, although such models are limited to large-enough plasma volumes where differentiability and integrability can be applied [3-5]. The differential methods become unsuccessful when considering the physical reality having nonintegral and nondifferentiable dynamics, for example, instabilities of complex systems that are generating chaos or patterns. Therefore, it is required to define explicitly a scale resolution in the expression of variables and thus implicitly in the equations governing the systems' evolution. Consequently, the dynamic variables remain space and time dependent as in the classical description, but they become also dependent on the scale resolution. Otherwise, instead of using nondifferentiable functions, one can work with various approximations of them, obtained by averaging at various scale resolutions. Therefore, dynamic variables behave as the limit of a family of functions, which at zero scale resolution are nondifferentiable, while at nonzero scale resolution are differentiable [6-8].

The previous approximation is well applied for complex system dynamics, because the real measurements are done for a finite scale resolution. This implies building a physical theory for a new geometric structure, by introducing the scale laws (and thus the scale transformation invariance) into the motion laws (which already are invariant to transformations of space and time coordinates). These requirements are satisfied by the scale relativity theory (SRT) [6] and more recently by the SRT in an arbitrary constant fractal dimension [7], where the interaction complexity is replaced by nondifferentiability, and motions take place without constraints 
on continuous but nondifferentiable curves in a fractal space time. Otherwise, when the time scale resolution is large enough when compared with the inverse value of the highest Lyapunov exponent $[9,10]$, potential routes are replacing deterministic trajectories and positions characterized by probability densities are substituting the definite ones. Therefore, the motion curves are both geodesics (in a fractal space) and fractal fluid stream lines, so that the geodesics are selected through the external constraints.

When the scale covariance principle is functional, the transition from differentiable to fractal physics is achieved by replacing the usual time derivative with the fractal operator [7]

$$
\frac{\widehat{d}}{d t}=\partial_{t}+\widehat{V}^{l} \partial_{l}-i \lambda(d t)^{\left(2 / D_{F}\right)-1} \partial^{l} \partial_{l}, \quad l=1,2,3,
$$

with

$$
\begin{aligned}
\partial_{t} & =\frac{\partial}{\partial t}, \\
\partial_{l} & =\frac{\partial}{\partial X_{l}}, \\
\partial_{l} \partial^{l} & =\frac{\partial}{\partial X_{l}}\left(\frac{\partial}{\partial X_{l}}\right), \\
\widehat{V}^{l} & =V_{D}^{l}-i V_{F}^{l}, \quad i=\sqrt{-1}, l=1,2,3,
\end{aligned}
$$

where $X_{l}$ is the fractal space coordinate, $t$ is the nonfractal time coordinate having the role of an affine parameter, and $\widehat{V}^{l}$ is the complex velocity field, of real part $V_{D}^{l}$, which is scale resolution $(d t)$ independent, and imaginary part $V_{F}^{l}$, which depends on the scale resolution. The diffusion coefficient, $\lambda$, describes the fractal-nonfractal transition. For the fractal dimension $D_{F}$ of the motion curves, one can choose various definitions (Kolmogorov, Hausdorff-Besikovitch, etc. $[9,10])$, but its value has to be kept constant during the analysis of the complex system dynamics.

The fractal operator (1) behaves as a scale covariant derivative, and it allows us to obtain the dynamics fundamental equations, similarly as in the differentiable case. Thus, applying the fractal operator (1) to the complex velocity field (4), it gives

$$
\frac{\widehat{d} \widehat{V}^{i}}{d t}=\partial_{t} \widehat{V}^{i}+\widehat{V}^{l} \partial_{l} \widehat{V}^{i}-i \lambda(d t)^{\left(2 / D_{F}\right)-1} \partial^{l} \partial_{l} \widehat{V}^{i}=0,
$$

while for an external scalar potential $U$, it results with

$$
\frac{\widehat{d} \widehat{V}^{i}}{d t}=\partial_{t} \widehat{V}^{i}+\widehat{V}^{l} \partial_{l} \widehat{V}^{i}-i \lambda(d t)^{\left(2 / D_{F}\right)-1} \partial^{l} \partial_{l} \widehat{V}^{i}=-\nabla U .
$$

In the present paper, by means of (4), new solutions of fractal velocity fields are obtained in the hypothesis of constant density, null fractal force, and constant flux momentum per length unit. Then, the theoretical results are applied in the field of laser-produced plasma, to explain various expansion features of carbon plasma obtained by excimer laser ablation in various focusing conditions. Such transient plasmas play a significant role in the production of various nanostructured materials (carbon nanotubes, nanowires, graphene, etc.) [11-13], and controlling their properties (expansion velocities, density, and temperature) allows a significant enhancement of the deposition process [14-16].

\section{Theoretical Aspects}

Separating the dynamics of the complex system on the resolution scale (on the differential one through the real part, $V_{D}^{l}$, and on the fractal one through the imaginary part, $V_{F}^{l}$ ) leads to

$\frac{\widehat{d} V_{D}^{i}}{d t}=\partial_{t} V_{D}^{i}+V_{D}^{l} \partial_{l} V_{D}^{i}-\left[V_{F}^{l}+\lambda(d t)^{\left(2 / D_{F}\right)-1} \partial^{l}\right] \partial_{l} V_{F}^{i}=0$,
$\frac{\widehat{d} V_{F}^{i}}{d t}=\partial_{t} V_{F}^{i}+V_{D}^{l} \partial_{l} V_{F}^{i}-\left[V_{F}^{l}+\lambda(d t)^{\left(2 / D_{F}\right)-1} \partial^{l}\right] \partial_{l} V_{D}^{i}=0$.

At differential resolution scale, this separation implies the fractal force,

$$
F_{F}^{i}=\left(V_{F}^{l}+\lambda(d t)^{\left(2 / D_{F}\right)-1} \partial^{l}\right) \partial_{l} V_{F}^{i}
$$

Nullifying its value,

$$
\left(V_{F}^{l}-\lambda(d t)^{\left(2 / D_{F}\right)-1} \partial^{l}\right) \partial_{l} V_{F}^{l}=0
$$

in the condition,

$$
\partial_{l} V_{F}^{i}=0
$$

induces a particular velocity field of explicit form given in the following.

Finding the solutions for these equations can be relatively difficult, due to the fact that this equation system is nonlinear $[4,5]$. However, for the particular case of a stationary flow in an $(x, y)$ symmetry plane, an analytical solution of this system exists. With the previous assumption, (8) and (9) take the form

$$
\begin{aligned}
V_{x} \frac{\partial V_{x}}{\partial x}+V_{y} \frac{\partial V_{x}}{\partial x} & =-\lambda(d t)^{\left(2 / D_{F}\right)-1} \frac{\partial^{2} V_{x}}{\partial y^{2}} \\
\frac{\partial V_{x}}{\partial x}+\frac{\partial V_{y}}{\partial y} & =0
\end{aligned}
$$

where $V_{F x}=V_{x}(x, y)$ and $V_{F y}=V_{y}(x, y)$ are the velocities along the $O x$ and $O y$ axes, respectively.

Let us impose the boundary conditions of the flow

$$
\begin{array}{r}
\lim _{y \rightarrow 0} V_{y}(x, y)=0, \\
\lim _{y \rightarrow 0} \frac{\partial V_{x}}{\partial y}=0, \\
\lim _{y \rightarrow \infty} V_{x}(x, y)=0,
\end{array}
$$


and that the flux momentum per length unit is constant,

$$
\Theta=\rho \int_{-\infty}^{+\infty} V_{x}^{2} d y=\text { const. }
$$

Such conditions imply that on the limit $y=0$, the velocity is directed on the $x$-axis and that it is on the $y$-axis at large distances, while (12) is the conservation law of a specific kinetic energy density along the $x$-axis.

Using the method from $[4,5]$, the following solutions result with

$$
\begin{aligned}
& V_{x}=\frac{\left[1.5(\Theta / 6 \rho)^{2 / 3}\right]}{\left[\lambda(d t)^{\left(2 / D_{F}\right)-1} x\right]^{1 / 3}} \cdot \sec ^{2} \frac{\left[(0.5 y)(\Theta / 6 \rho)^{1 / 3}\right]}{\left[\lambda(d t)^{\left(2 / D_{F}\right)-1} x\right]^{2 / 3}}, \\
& V_{y}=\frac{\left[4.5(\Theta / 6 \rho)^{2 / 3}\right]}{\left[3 \lambda(d t)^{\left(2 / D_{F}\right)-1} x\right]^{1 / 3}} \cdot\left[\frac{y(\Theta / 6 \rho)^{1 / 3}}{\left[\lambda(d t)^{\left(2 / D_{F}\right)-1} x\right]^{2 / 3}} \cdot \operatorname{sech}^{2} \frac{\left[(0.5 y)(\Theta / 6 \rho)^{1 / 3}\right]}{\left[\lambda(d t)^{\left(2 / D_{F}\right)-1} x\right]^{2 / 3}}-\tanh \frac{\left[(0.5 y)(\Theta / 6 \rho)^{1 / 3}\right]}{\left[\lambda(d t)^{\left(2 / D_{F}\right)-1} x\right]^{2 / 3}}\right] .
\end{aligned}
$$

For $y=0$, we obtain in (13) the critical flow velocity (maximum value along the $x$-axis) in the form

$$
V_{x}(x, y=0)=V_{c}=\frac{\left[1.5(\Theta / 6 \rho)^{2 / 3}\right]}{\left[\lambda(d t)^{\left(2 / D_{F}\right)-1} x\right]^{1 / 3}},
$$

while (12), taking into account (15), becomes

$$
\Theta=\rho \int_{-\infty}^{+\infty} V_{x}^{2}(x, y) d y=\rho \int_{-d_{c}}^{+d_{c}} V_{c}^{2}(x, 0) d y,
$$

so that the critical cross section of the stream line tube (diameter of the flowing tube corresponding to the critical flow velocity) of the complex system flow is given by

$$
d_{c}(x, y=0)=\frac{\Theta}{2 \rho V_{c}^{2}}=2.42\left[\lambda(d t)^{\left(2 / D_{F}\right)-1} x\right]^{2 / 3}\left(\frac{\rho}{\Theta}\right)^{1 / 3} .
$$

Equations (13), (14), and (15) can be significantly simplified by introducing the normalized quantities,

$$
\begin{aligned}
\xi & =\frac{x}{x_{0}}, \\
\eta & =\frac{y}{y_{0}}, \\
u & =V_{x} \frac{y_{0}^{2}}{x_{0} \lambda}, \\
v & =V_{y} \frac{y_{0}^{2}}{x_{0} \lambda}, \\
u_{c} & =V_{c} \frac{y_{0}^{2}}{x_{0} \lambda}, \\
\frac{(\Theta / 6 \rho)^{1 / 3}}{\lambda^{2 / 3}} & =\frac{x_{0}^{2 / 3}}{y_{0}},
\end{aligned}
$$

$$
\mu=\lambda(d t)^{\left(2 / D_{F}\right)-1},
$$

where $x_{0}, y_{0}$ are the specific lengths, $V_{c}$ is the specific velocity, and $\mu$ is the fractalization degree (similar to the one defined in [17]). It results that

$$
\begin{aligned}
u(\xi, \eta)= & \frac{1.5}{\xi^{1 / 3} \mu^{1 / 3}} \operatorname{sech}^{2}\left(\frac{0.5 \eta}{\xi^{2 / 3} \mu^{2 / 3}}\right), \\
v(\xi, \eta)= & \frac{4.5^{2 / 3}}{3^{1 / 3} \xi^{1 / 3} \mu^{1 / 3}} \\
& \cdot\left[\frac{\eta}{\xi^{2 / 3} \mu^{2 / 3}} \operatorname{sech}^{2}\left(\frac{0.5 \eta}{\xi^{2 / 3} \mu^{2 / 3}}\right)-\tanh \left(\frac{0.5 \eta}{\xi^{2 / 3} \mu^{2 / 3}}\right)\right], \\
u_{c}= & \frac{1.5}{\xi^{1 / 3} \mu^{1 / 3}} .
\end{aligned}
$$

Relation (20) suggests that, independent of the fractalization degree, the fractal velocity field is highly nonlinear: along the $(O \xi)$ flow axis, it is of soliton type $\left(\operatorname{sech}^{2}\right)$, while along the $(\mathrm{O} \eta)$ flow axis, it is a "mixture" between a soliton type, a kink type ( $\tanh )$, and so on $[4,18]$. The 3D dependences of the normalized components of the fractal velocity field on the nondimensional spatial coordinates are plotted in Figures 1(a) and $1(\mathrm{~b})$, along with its norm $V(\xi, \eta)=\sqrt{u(\xi, \eta)^{2}+v(\xi, \eta)^{2}}$ (Figure 1(c)), for the fractalization degree $\mu=1$. It results with a central symmetry axis of the flow $(O \xi)$ and a decrease of the velocity norm from the left boundary. Meanwhile, the local central maximum is adjacent with two symmetrical regions of the minimum.

In Figures 2(a)-2(d), the flow velocity field of the fractal fluid is given for various values of the $\mu$ parameter. One can observe that for low values of $\mu$, a $\mathrm{V}$-shaped fluid flow exists, having two lateral branches characterized by a "quasi-stagnating" structure (decreased values of the velocity 


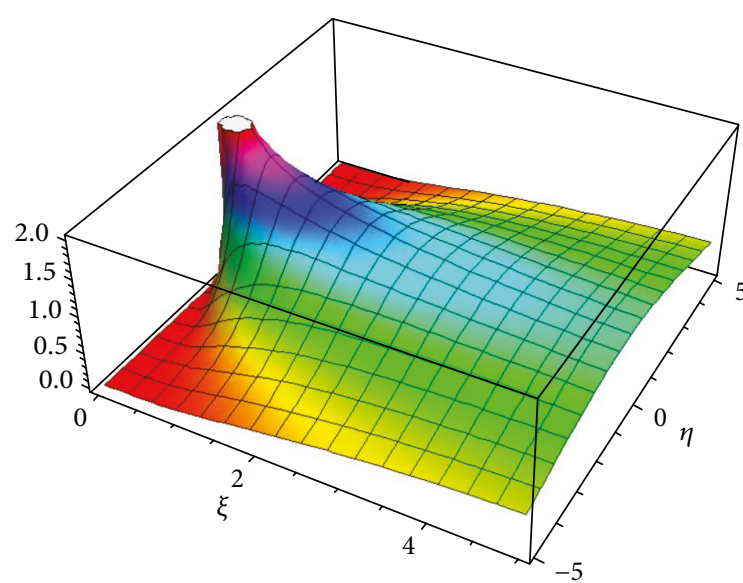

(a) $u(\xi, \eta)$

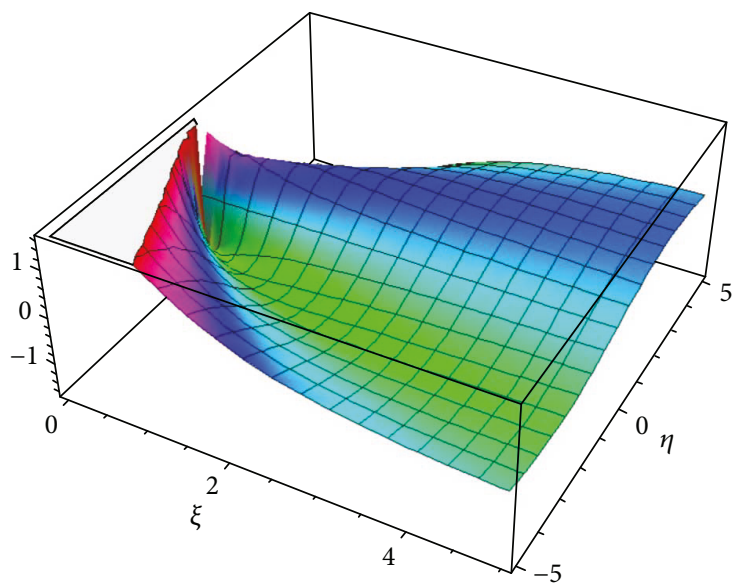

(b) $v(\xi, \eta)$

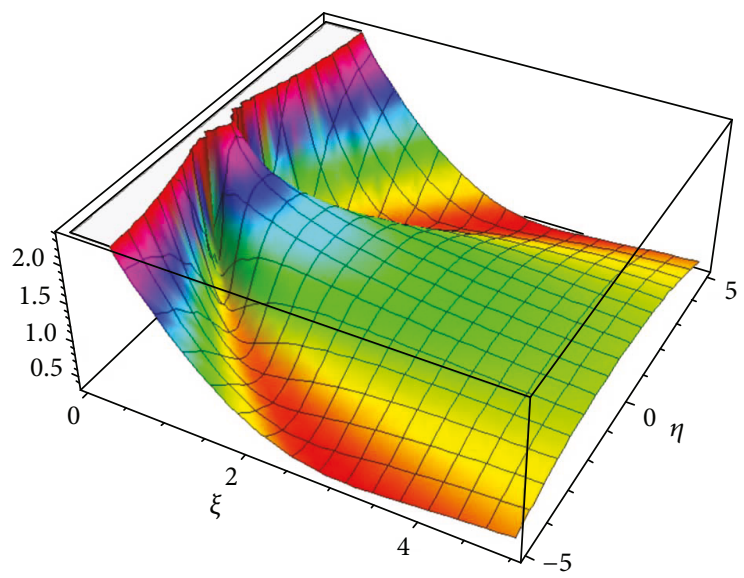

(c) $V(\xi, \eta)$

Figure 1: Space dependences of the normalized fractal velocity field components (a, b) and its norm (c), as given by (20) for $\mu=1$.

norm-see the arrow marks in Figure 2(a)). Meanwhile, in the central part, the speed has its local maximum value, regardless of the values of the $\mu$ parameter.

In the following, these theoretical results are applied to explain the expansion of laser-produced carbon plasma in various laser beam-focusing conditions.

\section{Experimental Aspects}

The experimental setup used for measurements is described in details in [19-21]. Briefly, carbon plasma has been produced by the ablation of high-purity (99.99\%) pyrolytic graphite targets placed in an evacuated chamber $(0.0001 \mathrm{~Pa})$ using a $\mathrm{KrF}$ excimer laser $(248 \mathrm{~nm}, 20 \mathrm{~ns}$, and $5 \mathrm{~Hz})$. The beam was directed onto the target surface at a $45^{\circ}$ incidence angle and focused through a spherical lens of a $500 \mathrm{~mm}$ focal length. The laser energy was typically set to $E_{L}=200 \mathrm{~mJ} /$ pulse. Before focusing, the beam profile had approximately a rectangular geometry, with a top-hat energy distribution for the long axis ( $24 \mathrm{~mm}$ characteristic size) and a Gaussian one for the short axis $(10 \mathrm{~mm}$ full width at half maximum). The laser fluence was varied in a moderate regime, below $3 \mathrm{~J} / \mathrm{cm}^{2}$, by changing the distance between the focusing lens and the target surface, further denoted by the lens position (LP). Using the spot area on the target, we estimated fluence values of $1.6 \mathrm{~J} / \mathrm{cm}^{2}$ for $\mathrm{LP}=46 \mathrm{~cm}$ and $0.9 \mathrm{~J} / \mathrm{cm}^{2}$ for $\mathrm{LP}=41 \mathrm{~cm}$ (for the sake of simplicity, only the LP values will be displayed in the following). The global (i.e., not spectrally dispersed) plasma emission at different delays with respect to the laser pulse was recorded by ICCD fast imaging (Andor iStar DH740-18U-03 camera) in side view. Each image corresponds to a single laser pulse and 20 ns ICCD camera gate time. For ion current measurements, a Faraday cup (FC) was placed on the axis of the ablation plume or circularly displaced in various angular positions with respect to this axis, preserving the same distance of $14 \mathrm{~cm}$ from the center of the laser impact spot on the target surface. A $5 \mathrm{~mm}$ diameter aperture was used at the entrance of the FC, to narrow the ion collecting area and to achieve a better spatial resolution. A voltage of $-30 \mathrm{~V}$ applied on the FC was determined to be sufficient for repelling the plasma electrons, in order to record the ion saturation current. The electrical signals were recorded by using the $50 \Omega$ input impedance of a $2 \mathrm{GHz}$ oscilloscope (LeCroy), externally triggered by a fast-response signal photodiode ( $2 \mathrm{~ns}$ rise time). Each recorded time-of-flight (TOF) signal was obtained from a single laser pulse directed onto fresh surfaces of the target. 


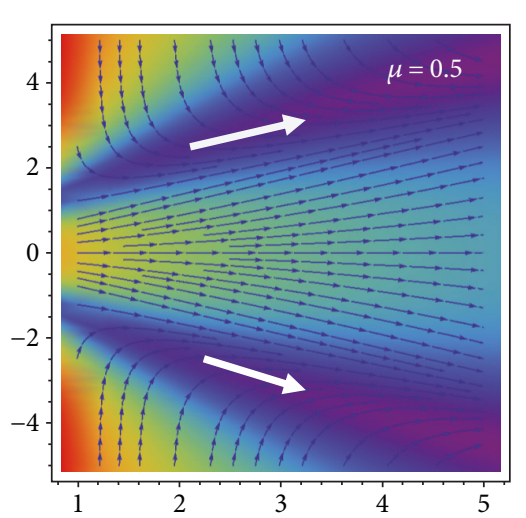

(a)

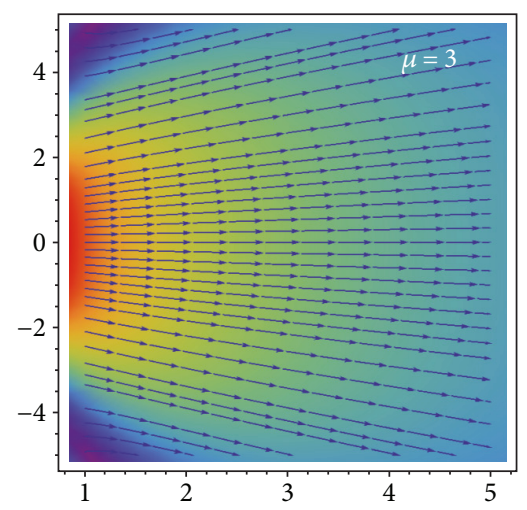

(c)

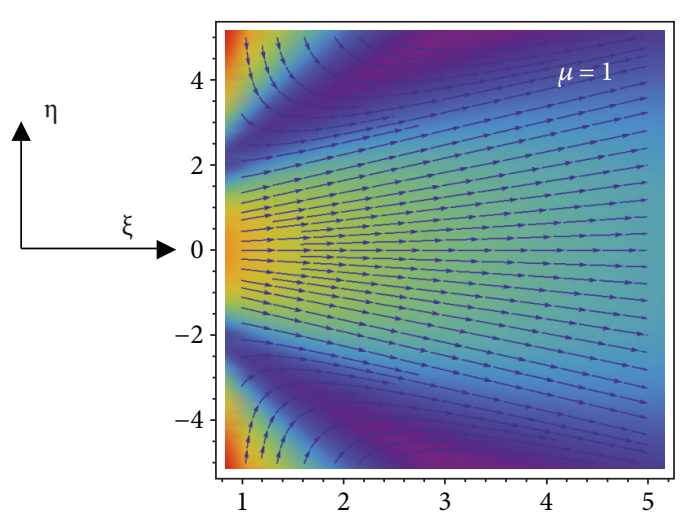

(b)

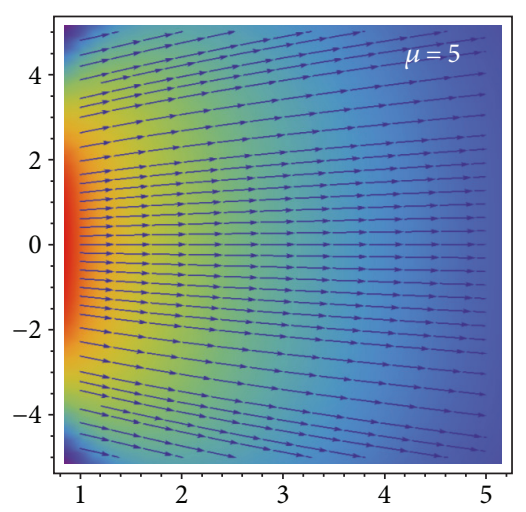

(d)

FIgURE 2: Flow velocity field as given by (20) for various $\mu$ values.

In Figure 3(a), the time evolution of plasma images recorded for $\mathrm{LP}=46 \mathrm{~cm}$ shows the existence of a V-like radiating shape, having two lateral arms (with an angle of about $30^{\circ}$ ) that originate from two "seeds" of plasma observed after $300 \mathrm{~ns}$ from the laser pulse. The central part contains faster particles expanding along the plume axis with higher velocity, which are hardly discernible in a region of decreased contrast, for example, at 2000 ns (see dotted red marks in Figure 3(a)). We note that for each image, relative intensities (with respect to the maximum recorded value) were used, to better observe the radiating features.

Such peculiar plasma shape is significantly influenced by the laser beam focusing on the target. In Figure 3(b), at $500 \mathrm{~ns}$ after the laser pulse, that is, the time sequence considered to be most relevant for subsequent plasma dynamics, one can observe that for $\mathrm{LP}=41 \mathrm{~cm}$ and $\mathrm{LP}=42 \mathrm{~cm}$, the usual "ellipsoidal" plume shape was recorded, that is in agreement with the "top-hat" energy distribution for the long axis of the laser beam [22]. The V-like shape becomes observable at $\mathrm{LP}=44 \mathrm{~cm}$, while for $\mathrm{LP}=46 \mathrm{~cm}$, the two plasma "seeds" appear to be completely separated. In previous works $[20,21]$, we observed some correlations with the ablation crater depth profile. Thus, for $\mathrm{LP}=41 \mathrm{~cm}$ and $\mathrm{LP}=42$, its shape is mirroring the laser energy distribution, but for $\mathrm{LP}=46 \mathrm{~cm}$, two ablation depths were observed, with a deeper hole in the crater center and two lateral thresholds at distances similar with the long axis of the laser beam $[20,21]$.
When plasma expansion takes place in an ambient gas (Ar, $1 \mathrm{~Pa}$ pressure), the well-known drag effect [23-26] can be observed, as it results (from Figure 3(c)) in various focusing conditions at $2000 \mathrm{~ns}$ delay. The lateral arms of the V-shaped transform in two radiating "balls," flying almost parallel after $2000 \mathrm{~ns}$.

Using the FC as ion collector in various angular positions $(\theta)$ with respect to the plasma expansion central axis (i.e., the normal to the target surface in the laser impact spot) shows that the maximum number of charged particles is always recorded on the normal direction to the target, regardless of the laser beam focusing (Figures 4(a)-4(c)). Such result is unexpected for $\mathrm{LP}=46 \mathrm{~cm}$, where most of the recorded optical radiation comes from the lateral arms. However, in the central regions, prompt and low-emitting particles were previously distinguished that can explain the FCrecorded current.

Such fast particles are better evidenced in the presence of a deposition substrate located at $50 \mathrm{~mm}$ in front of the target. In Figure 5, we extracted the emission intensities along the $z$-axis (normal to the target surface in the laser spot center) by cross-sectioning ICCD images (like the ones in Figure 3) at various delays with respect to the laser pulse (for $\mathrm{LP}=46 \mathrm{~cm}$ and a fluence of $2.1 \mathrm{~J} / \mathrm{cm}^{2}$ ). One can observe that fast particles accumulate in front of the substrate starting with $1500 \mathrm{~ns}$, which roughly means a maximum of expansion velocity $v_{\text {fast }}=3 \cdot 1 \cdot 10^{4} \mathrm{~m} / \mathrm{s}$. They are followed by a slower 


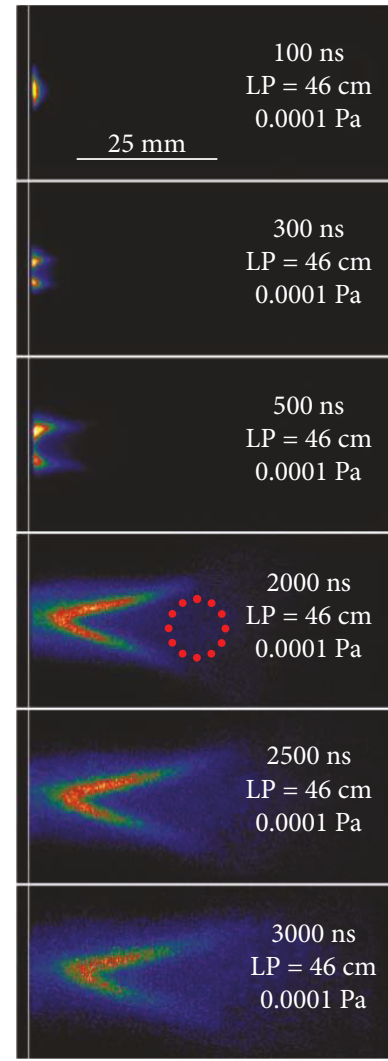

(a)

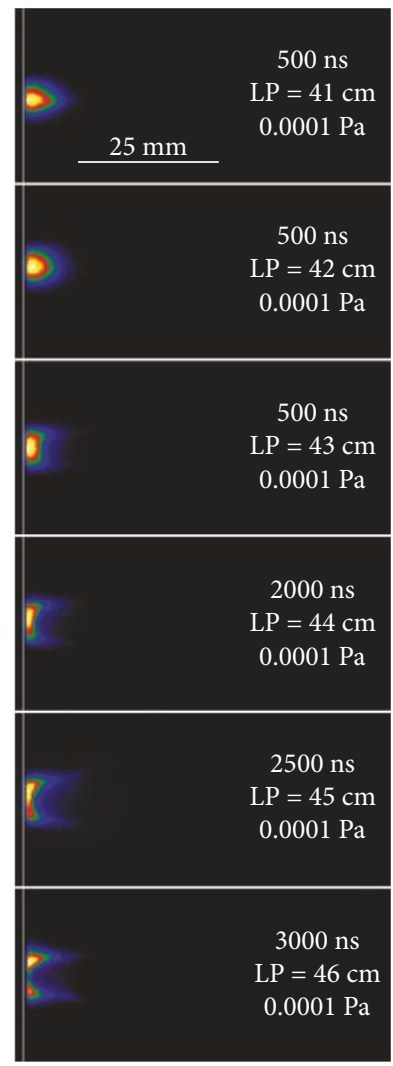

(b)

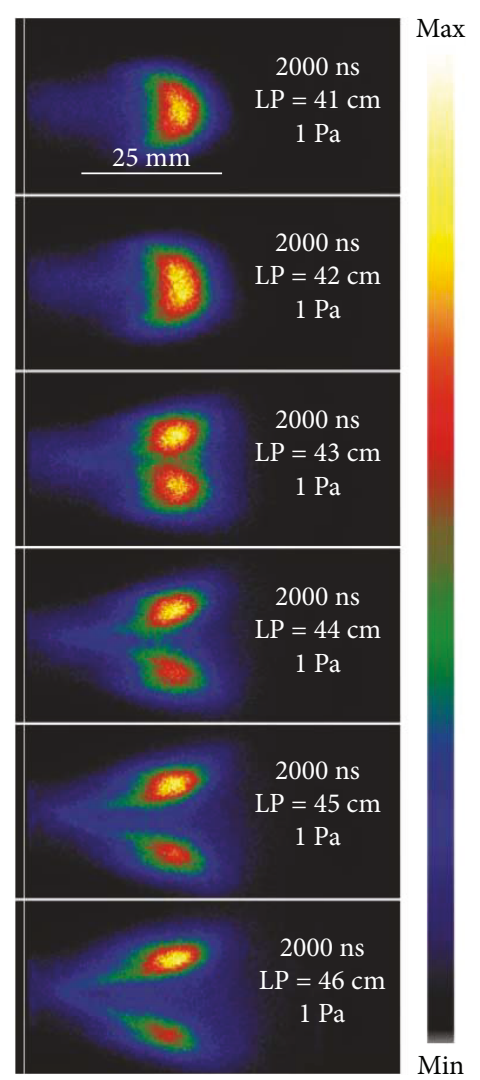

(c)

FIGURE 3: Images of the V-like plasma shape obtained for $\mathrm{LP}=46 \mathrm{~cm}$ at various stages of time evolution (a), comparison between visible radiating shapes obtained for various laser beam focusing at $500 \mathrm{~ns}$ delay (b), and drag effect observed for expansion in ambient gas (c).

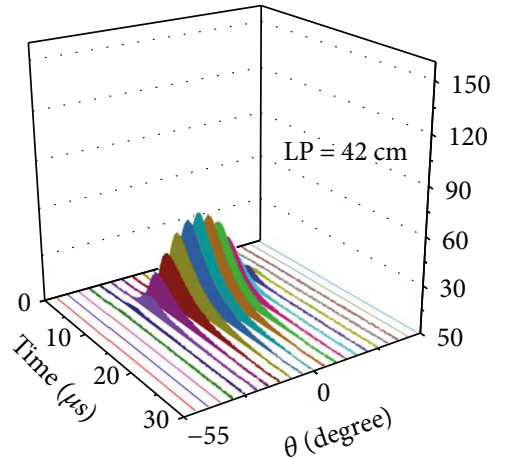

(a)

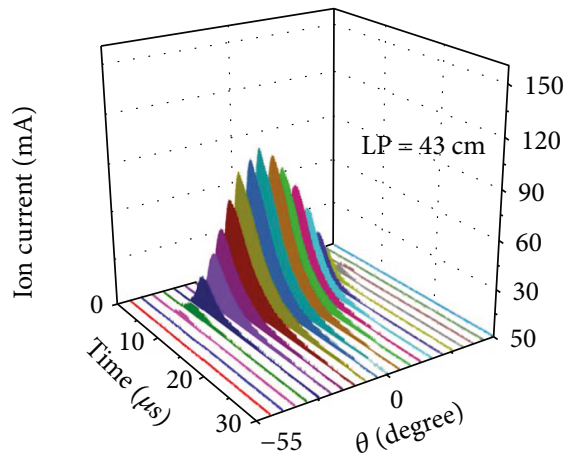

(b)

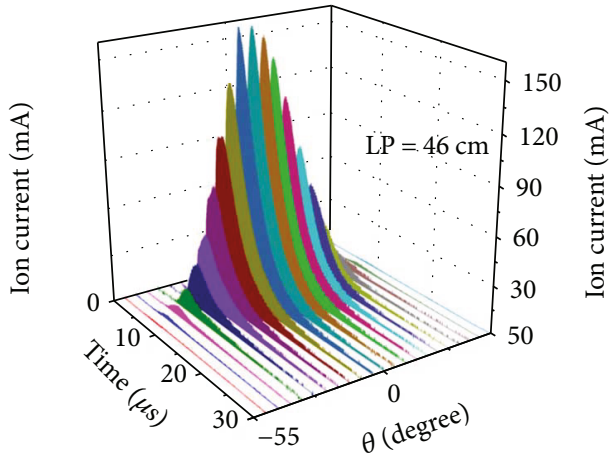

(c)

FIGURE 4: Temporal traces of FC-recoded signals in various angular positions and different laser beam focusing $\mathrm{LP}=42 \mathrm{~cm}(\mathrm{a}), \mathrm{LP}=43 \mathrm{~cm}(\mathrm{~b})$, and $\mathrm{LP}=46 \mathrm{~cm}(\mathrm{c})$.

structure, emitted through a thermal mechanism [27-30] from the target. For this part, the dependence of the maximum-emitting positions versus time delays (dashed line in Figure 5) resulted to be linear and the derived "slow" structure velocity $\left(v_{\text {slow }}=3.3 \cdot 10^{3} \mathrm{~m} / \mathrm{s}\right)$ is one order of magnitude lower than the "fast" structure one.

Returning now to the theoretical results given by (20) and plotted in Figures 2(a)-2(d), let us observe a good agreement with plasma images given in Figure 3(b). The fractalization degree used in the model can be correlated with the collision rate in the ablation plasma, in the sense that increased collision rates correspond to low values of fractalization $(\mu)$. According to (19), the parameter $\mu$ is connected with the "fractal" contribution, by means of fractal-nonfractal transition coefficient, $\lambda$, and scale resolution, $d t$, and fractal dimension, $D_{F}$, of the movement curves. Between two successive 


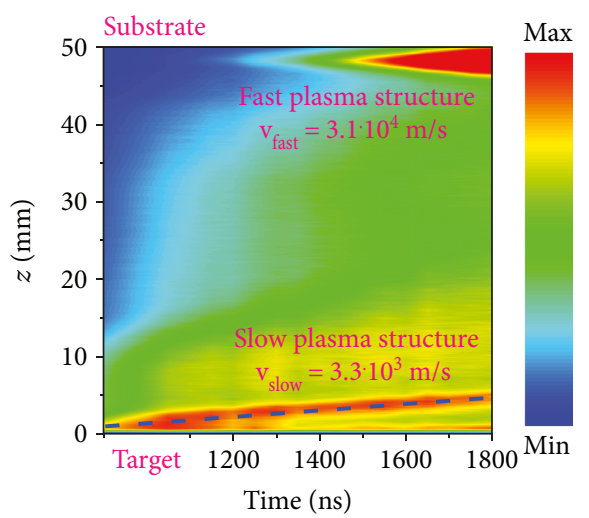

FiguRE 5: Excimer laser-produced carbon plasma expansion in vacuum: space-time contour plot of emission intensity along the $z$-axis ( $\mathrm{LP}=46 \mathrm{~cm}, 2.1 \mathrm{~J} / \mathrm{cm}^{2}$ fluence).

collisions in the ablation plasma, the trajectory of any particle is a straight line, that is, a continuous and differentiable curve, while in the impact point, it becomes nondifferentiable. Then, assuming that all the collision impact points are defining an uncountable set of points, it results that the trajectories become continuous and nondifferentiable curves. In such conjecture, the more collisional plasma means more broken trajectories, that is, an increased fractal dimension, $D_{F}$, and consequently a decreased fractalization degree $\mu$. From an experimental point of view, tighter focusing of the laser beam (i.e., higher LP in Figure 3(b)) leads to a denser ablation plasma, that is, more collisions between particles, which explains the very good qualitative agreement between Figure 3(b) (experiment) and Figure 2 (theory). We note that in [17], a similar interpretation of the fractalization degree was able to explain the well-known fast (hot) and slow (cold) plasma structures ejected through electrostatic and thermal mechanisms, respectively.

The V-shaped lateral arms of the expanding carbon plasma obtained for $\mathrm{LP}=46 \mathrm{~cm}$ and $\mathrm{LP}=45 \mathrm{~cm}$ correspond to "quasi-stagnating" regions of intense optical radiation emission. Our preliminary spectroscopic investigations show the dominance in these regions of carbon dimer vibrational transitions. It is known that the presence of the $\mathrm{C}_{2}$ molecule in the plasma plume favors the nanoparticle and cluster production $[31,32]$. There are two mechanisms assumed as responsible for dimer formation: direct ejection from the target and three-body recombination [33, 34]. In our opinion, the existence of the theoretically predicted and experimentally evidenced quasi-stagnating regions promotes the second mechanism in the enhancement of $\mathrm{C}_{2}$ molecule content. These investigations are underway and will be published in a separate paper.

\section{Conclusions}

A fractal approach is proposed to simulate peculiar dynamics of carbon plasmas generated by excimer laser ablation, by substituting particle motions on continuous and differential curves in a Euclidean space with free motions on continuous and nondifferentiable (fractal) curves in a fractal space. The mathematical procedure imposes the use of a motion operator, having the role of scale covariant derivative and then obtaining the fractal space geodesics by postulating a principle of scale covariance. For plane symmetry, stationary geodesic equations at a fractal scale resolution give a fractal velocity field with components expressed by means of nonlinear solutions (soliton type, kink type, etc.).

The theoretical results are applied in the field of laserproduced plasma, more precisely to explain various dynamics of carbon plasma obtained by excimer laser ablation in different focusing conditions. The occurrence of a V-shaped radiating plasma structure, consisting in two lateral stagnating arms of high emissivity and a fast-expanding central part of low emissivity, is evidenced, both experimentally and theoretically. Such peculiar plasma shape results for specific values of the fractalization degree which are correlated with the ablation laser-focusing conditions. The more collisional plasma means more broken trajectories, that is, an increased fractal dimension and consequently a decreased fractalization degree. From the experimental point of view, tighter focusing of the laser beam leads to a denser ablation plasma, that is, more collisions between particles, which explains the very good qualitative agreement between experiment and theory.

\section{Data Availability}

The data used to support the findings of this study are available from the corresponding author upon request.

\section{Conflicts of Interest}

The authors declare that they have no conflicts of interest.

\section{Acknowledgments}

Part of the present work was supported by the Romanian Space Agency (ROSA) within the Space Technology and Advanced Research (STAR) Program (Project no. 169/ 20.07.2017).

\section{References}

[1] R. Badii and A. Politi, Complexity: Hierarchical Structures and Scaling in Physics, Cambridge University Press, Cambridge, 1997.

[2] M. Mitchell, Complexity: A Guided Tour, Oxford University Press, Oxford, 2009.

[3] O. D. Michel and B. G. Thomas, Mathematical Modelling for Complex Fluids Flows, Springer, New York, 2012.

[4] G. K. Batchelor, An Introduction to Fluid Dynamics, Cambridge University Press, Cambridge, 2000.

[5] L. D. Landau and E. M. Lifshitz, Fluid Mechanics, Butterworth Heinemann, Oxford, 2nd ed. edition, 1987.

[6] L. Nottale, Scale Relativity and Fractal Space-Time: A New Approach to Unifying Relativity and Quantum Mechanics, Imperial College Press, London, 2011.

[7] I. Merches and M. Agop, Differentiability and Fractality in Dynamics of Physical Systems, World Scientific Publishing, Singapore, 2015. 
[8] J. Cresson, "Non-differentiable deformations OF $\mathbb{R}^{\mathrm{n}}$," International Journal of Geometric Methods in Modern Physics, vol. 3, no. 7, pp. 1395-1415, 2006.

[9] B. B. Mandelbrot, The Fractal Geometry of Nature, WH Freeman and Co., New York, 1983.

[10] J. Federer and A. Aharoner, Fractals in Physics, NorthHolland, Amsterdam, 1990.

[11] D. H. Lowndes, D. B. Geohegan, A. A. Puretzky, D. P. Norton, and C. M. Rouleau, "Synthesis of novel thin-film materials by pulsed laser deposition," Science, vol. 273, no. 5277, pp. 898-903, 1996.

[12] L. Torrisi, F. Caridi, D. Margarone, A. Picciotto, A. Mangione, and J. J. Beltrano, "Carbon-plasma produced in vacuum by 532 nm-3 ns laser pulses ablation," Applied Surface Science, vol. 252, no. 18, pp. 6383-6389, 2006.

[13] F. Guzmán, M. Favre, H. M. Ruiz et al., "Pulsed laser deposition of thin carbon films in a neutral gas background," Journal of Physics D: Applied Physics, vol. 46, no. 21, article 215202, 2013

[14] A. A. Puretzky, D. B. Geohegan, X. Fan, and S. J. Pennycook, "Dynamics of single-wall carbon nanotube synthesis by laser vaporization," Applied Physics A: Materials Science \& Processing, vol. 70, no. 2, pp. 153-160, 2000.

[15] S. S. Harilal, R. C. Issac, C. V. Bindhu, V. P. N. Nampoori, and C. P. G. Vallabhan, "Optical emission studies of $\mathrm{C}_{2}$ species in laser-produced plasma from carbon," Journal of Physics D: Applied Physics, vol. 30, no. 12, pp. 1703-1709, 1997.

[16] M. Ruiz, F. Guzmán, M. Favre et al., "Characterization of a laser plasma produced from a graphite target," Journal of Physics Conference Series, vol. 511, article 012064, 2014.

[17] S. A. Irimiciuc, S. Gurlui, P. Nica, C. Focsa, and M. Agop, “A compact non-differential approach for modeling laser ablation plasma dynamics," Journal of Applied Physics, vol. 121, no. 8, article 083301, 2017.

[18] E. A. Jackson, Perspectives of Nonlinear Dynamics, Cambridge University Press, Cambridge, 1989.

[19] C. Ursu and P. E. Nica, "Diagnosis of carbon laser produced plasma by using an electrostatic energy analyzer," Journal of Optoelectronics and Advanced Materials, vol. 15, no. 1-2, pp. 42-45, 2013.

[20] P. E. Nica, G. B. Rusu, O. G. Dragos, and C. Ursu, "Effect of excimer laser beam spot size on carbon laser-produced plasma dynamics," IEEE Transactions on Plasma Science, vol. 42, no. 10 , pp. 2694-2695, 2014

[21] C. Ursu, P. Nica, and C. Focsa, "Excimer laser ablation of graphite: the enhancement of carbon dimer formation," Applied Surface Science, vol. 456, pp. 717-725, 2018.

[22] M. R. Perrone, S. Tundo, C. Panzera, and G. De Nunzio, "Beam-divergence control of excimers with plane-parallel Gaussian cavities," Applied Optics, vol. 36, no. 33, pp. 85748581, 1997.

[23] J. Schou, S. Amoruso, and J. G. Lunney, "Plume dynamics," in Laser Ablation and Its Applications, C. Phipps, Ed., vol. 129 of Springer Series in Optical Sciences, pp. 67-95, Springer, Boston, MA, 2007.

[24] A. K. Sharma and R. K. Thareja, "Characterization of laserproduced aluminum plasma in ambient atmosphere of nitrogen using fast photography," Applied Physics Letters, vol. 84, no. 22, pp. 4490-4492, 2004.

[25] S. S. Harilal, C. V. Bindhu, M. S. Tillack, F. Najmabadi, and A. C. Gaeris, "Internal structure and expansion dynamics of laser ablation plumes into ambient gases," Journal of Applied Physics, vol. 93, no. 5, pp. 2380-2388, 2003.

[26] D. B. Geohegan, "Fast intensified-CCD photography of $\mathrm{YBa}_{2}$ $\mathrm{Cu}_{3} \mathrm{O}_{7}$-xlaser ablation in vacuum and ambient oxygen," Applied Physics Letters, vol. 60, no. 22, pp. 2732-2734, 1992.

[27] C. Focsa, S. Gurlui, P. Nica, M. Agop, and M. Ziskind, "Plume splitting and oscillatory behavior in transient plasmas generated by high-fluence laser ablation in vacuum," Applied Surface Science, vol. 424, pp. 299-309, 2017.

[28] P. Nica, S. Gurlui, M. Osiac, M. Agop, M. Ziskind, and C. Focsa, "Investigation of femtosecond laser-produced plasma from various metallic targets using the Langmuir probe characteristic," Physics of Plasmas, vol. 24, no. 10, article 103119, 2017.

[29] C. Ursu, O. G. Pompilian, S. Gurlui et al., " $\mathrm{Al}_{2} \mathrm{O}_{3}$ ceramics under high-fluence irradiation: plasma plume dynamics through space- and time-resolved optical emission spectroscopy," Applied Physics A: Materials Science \& Processing, vol. 101, no. 1, pp. 153-159, 2010.

[30] C. Focsa, P. Nemec, M. Ziskind, C. Ursu, S. Gurlui, and V. Nazabal, "Laser ablation of $\mathrm{As}_{\mathrm{x}} \mathrm{Se}_{100-\mathrm{x}}$ chalcogenide glasses: plume investigations," Applied Surface Science, vol. 255, no. 10, pp. 5307-5311, 2009.

[31] M. K. Moodley and N. J. Coville, "Experimental evidence for the early nucleation of single-walled carbon nanotubes," Chemical Physics Letters, vol. 498, no. 1-3, pp. 140-144, 2010.

[32] A. Van Orden and R. J. Saykally, "Small carbon clusters: spectroscopy, structure, and energetics," Chemical Reviews, vol. 98 , no. 6 , pp. $2313-2358,1998$.

[33] K. Sasaki, T. Wakasaki, S. Matsui, and K. Kadota, "Distributions of $\mathrm{C}_{2}$ and $\mathrm{C}_{3}$ radical densities in laser-ablation carbon plumes measured by laser-induced fluorescence imaging spectroscopy," Journal of Applied Physics, vol. 91, no. 7, pp. 4033-4039, 2002.

[34] K. F. Al-Shboul, S. S. Harilal, and A. Hassanein, "Emission features of femtosecond laser ablated carbon plasma in ambient helium," Journal of Applied Physics, vol. 113, no. 16, article 163305, 2013. 


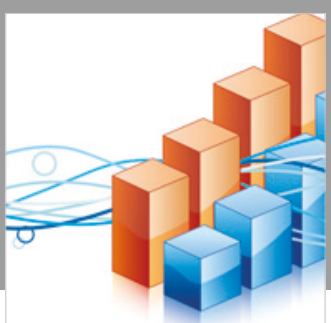

Advances in

Operations Research

\section{-n-m}
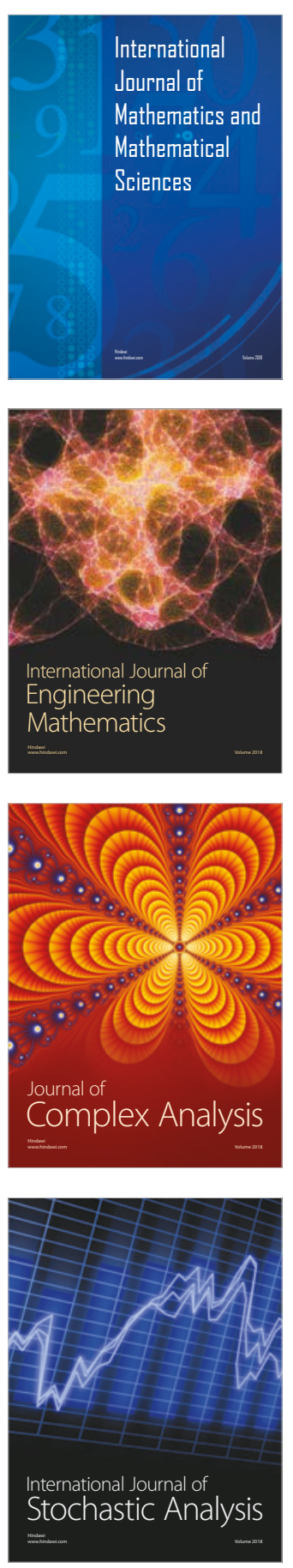
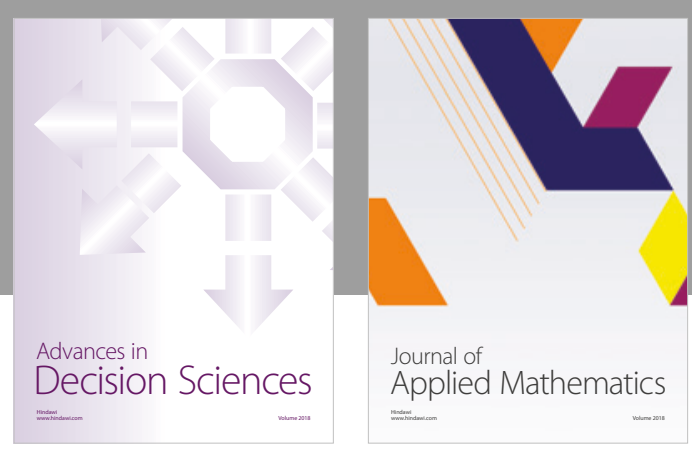

Journal of

Applied Mathematics
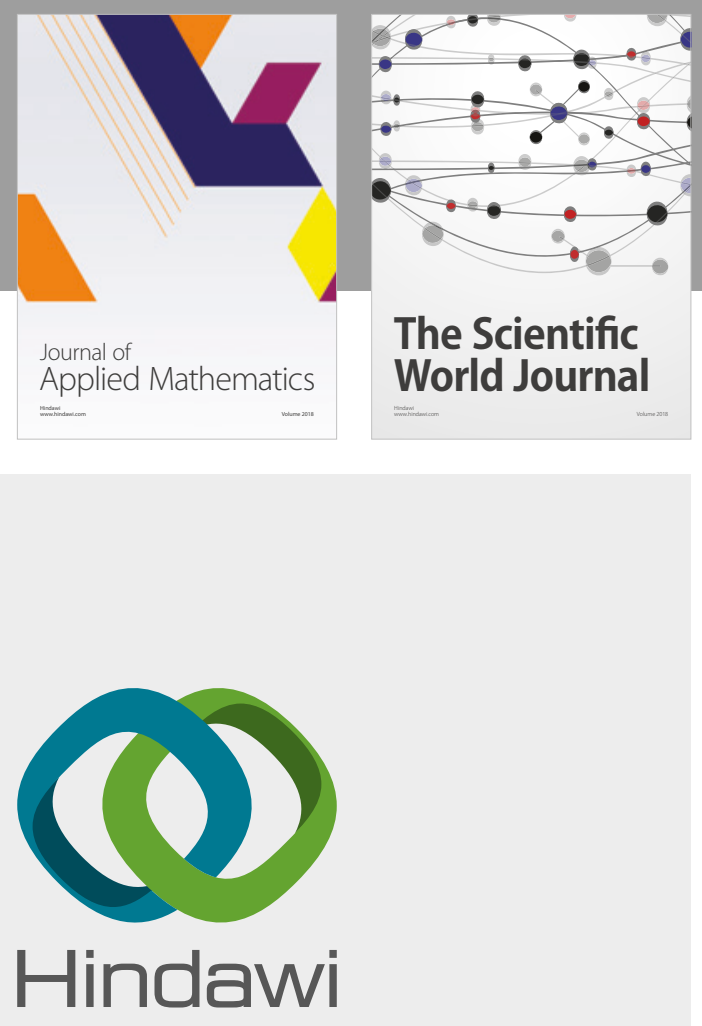

Submit your manuscripts at

www.hindawi.com

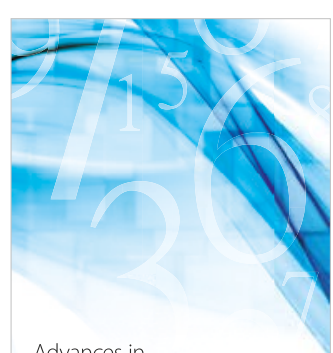

Advances in
Numerical Analysis
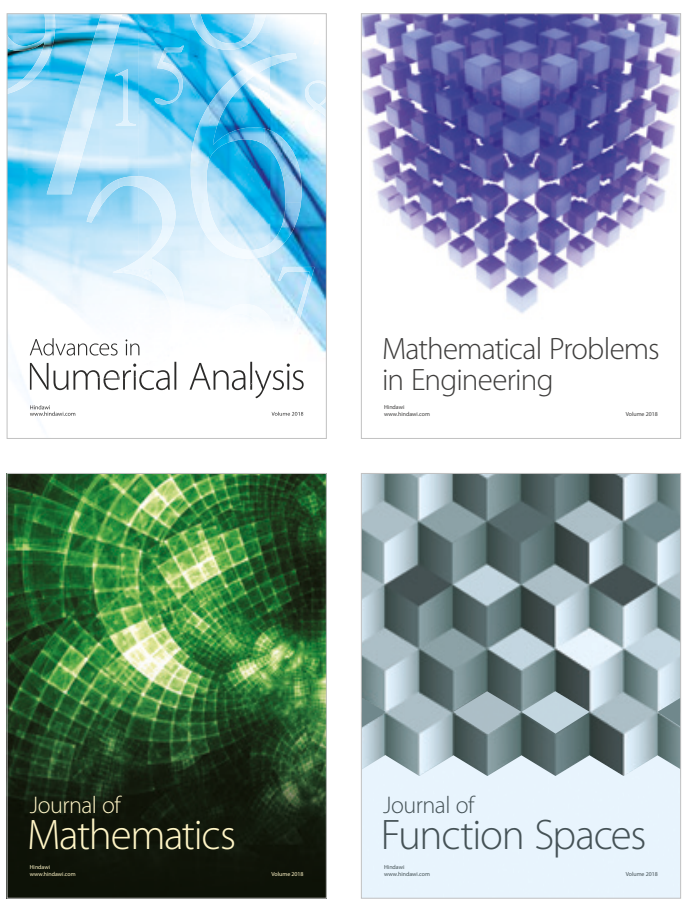

Mathematical Problems in Engineering

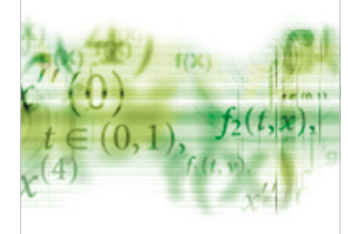

International Journal of

Differential Equations

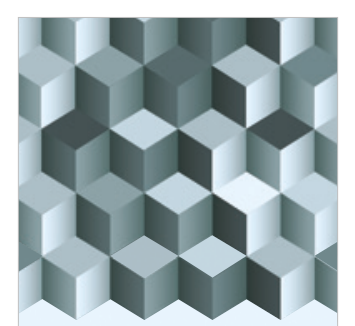

Journal of

Function Spaces
The Scientific

World Journal

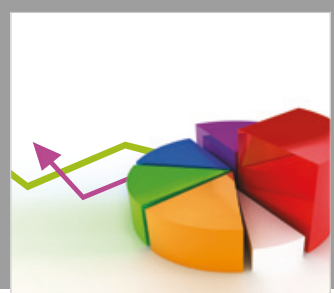

Journal of

Probability and Statistics
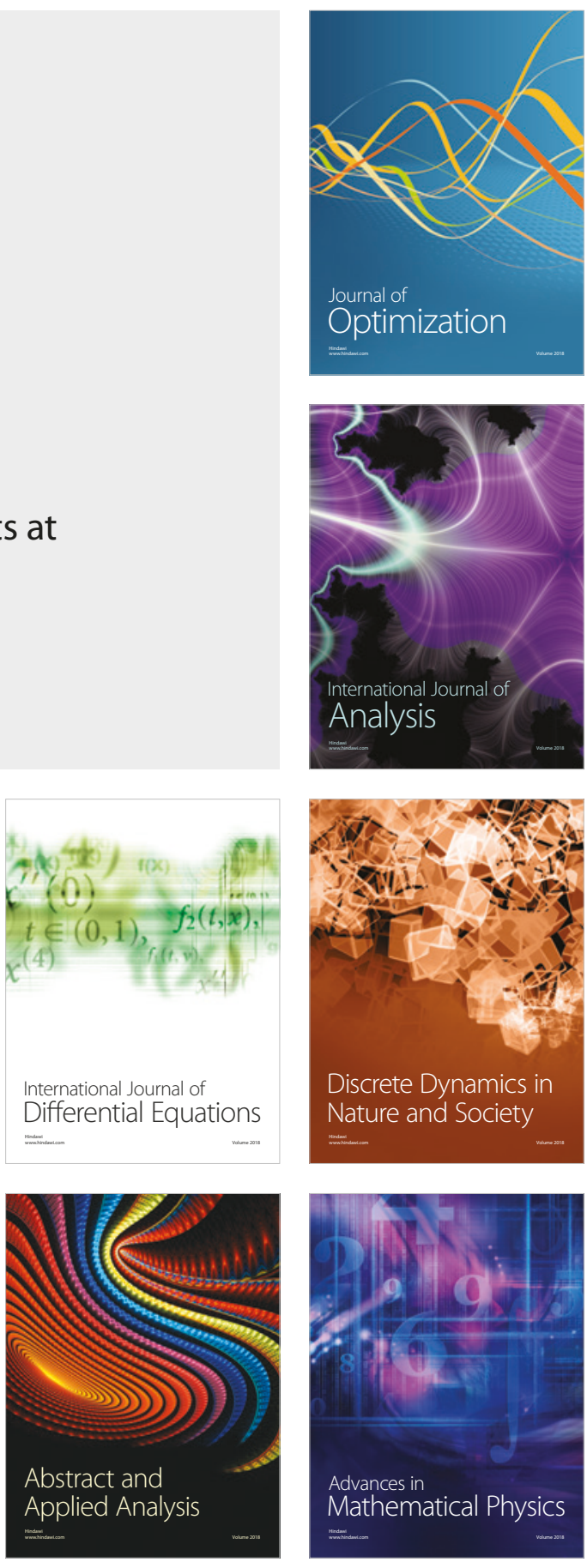